\title{
The Special Case of $\omega$ Centauri
}

\author{
John E. Norris \\ Research School of Astronomy $\mathcal{G}$ Astrophysics, Institute of Advanced \\ Studies, The Australian National University, Mount Stromlo \\ Observatory, Cotter Road, Weston ACT 2611, Australia
}

\begin{abstract}
Cen is unique among the Galactic globular clusters. It exhibits not only classical mixing signatures, but also those of primordial chemical enrichment by both massive and intermediate-mass stars: the abundances of all elements vary, not just those of the $\mathrm{C}, \mathrm{N}, \mathrm{O}, \mathrm{Na}, \mathrm{Mg}$, and Al. The challenge is to disentangle the roles of mixing and primordial phenomena. We discuss here what sets $\omega$ Cen apart from the other clusters.
\end{abstract}

\section{Introduction}

It has been clear since the publication of the first color-magnitude diagrams of $\omega$ Cen by Woolley et al. (1966, photographic) and Cannon \& Stobie (1973, photoelectric) that this system is special, and differs fundamentally from other Galactic globular clusters. The contrast between its wide and complicated giant branch and the narrow ones of all other clusters with accurate photometry and non-patchy reddening is stark. Two recent color-magnitude diagrams of the system show strong evidence for discrete substructure on the giant branch (Lee et al. 1999; Pancino et al. 2000).

As described by Kraft in this volume, most if not all other clusters show intracluster abundance variations of some subset of $\mathrm{C}, \mathrm{N}, \mathrm{O}, \mathrm{Na}, \mathrm{Mg}$, and $\mathrm{Al}$, arising from nucleosynthetic processes involving proton-capture reactions. There is, however, little if any evidence for variations in the $\alpha(\mathrm{Mg}$ excepted) and ironpeak elements, although small variations in the heavy neutron-capture elements appear to exist in at least one cluster. In these simpler systems the argument centers on how much can be explained in terms of evolutionary phenomena (deep mixing) not predicted by standard theoretical models and how much originates in effects primordial to the cluster (already existing when star formation ceased).

$\omega$ Cen is different. While it exhibits all of the phenomena seen in the other clusters, it shows others that are not, and which are naturally explained in terms of primordial effects. These differences, in the context of chemical abundances, will be highlighted here. (Their relationship to the kinematics within the system lies outside the terms of this review.) $\$ 2$ discusses the results of high resolution analyses that provide the basic information on the abundance patterns. $\S 3$ highlights recent investigations that constrain the enrichment history of the cluster. Finally, in $\S 4$ a brief discussion is given of an in-progress investigation of the abundance patterns on the cluster's main sequence. 


\section{Abundance patterns on the giant branch}

As noted above, $\omega$ Cen exhibits abundance variations among what one might refer to as the usual suspects (in particular $\mathrm{C}, \mathrm{N}, \mathrm{O}, \mathrm{Na}$, and $\mathrm{Al}$ ) as was shown, for example, by Norris \& Da Costa (1995a,b). Strong anticorrelations exist between $\mathrm{Na}$ and $\mathrm{Al}$ on the one hand and $\mathrm{O}$ on the other. An important difference, first appreciated by Persson et al. (1980), is that the behavior of $\mathrm{C}$ is more complicated than in other clusters: as well as containing $\mathrm{CH}$ stars, $\omega$ Cen has a subclass of objects with significantly enhanced carbon (which remains in need of comprehensive explanation).

The uniqueness of $\omega$ Cen lies in the fact that one finds a spread in the abundances of all elements. (See e.g. Norris \& Da Costa 1995b; Smith, Cunha \& Lambert 1995; Smith et al. 2000$)$. [ $\mathrm{Fe} / \mathrm{H}]$ varies over the range -1.8 to $\sim-0.5$. Relative abundances are instructive: $[\alpha / \mathrm{Fe}] \sim 0.2-0.5^{1}$ and [iron-peak $\left./ \mathrm{Fe}\right] \sim 0.0$, both consistent with primordial intracluster enrichment by massive supernovae. For these elements one sees within $\omega$ Cen the same behavior one finds within the Galactic globular cluster population as a whole. The situation for the heavy neutron-capture elements is even more interesting. Within $\omega \mathrm{Cen}$, [s-process/Fe] rises more rapidly as a function of $[\mathrm{Fe} / \mathrm{H}]$ that it does in the cluster population as a whole. The existence of s-process enhancement signals a relatively long period of cluster enrichment involving intermediate mass AGB stars, while the overabundance relative to $\mathrm{Fe}$ suggests a more prominent role of intermediate mass stars relative to more massive ones in the enrichment process.

The above results derive from analysis of a highly biased sample of some 40-50 red giants chosen to encompass the entire range of cluster peculiarities. The form of the abundance distribution of [metals $/ \mathrm{H}]$ is also instructive. From medium-resolution studies of complete samples of several hundred red giants, the following picture emerges. Few if any stars have $[\mathrm{Fe} / \mathrm{H}]<-2.0$. There is a well-defined peak in the distribution at $[\mathrm{Fe} / \mathrm{H}]=-1.8$, with a long tail stretching up to $[\mathrm{Fe} / \mathrm{H}] \sim-0.5$ (Norris, Freeman \& Mighell 1996; Suntzeff \& Kraft 1996). The former authors report the existence of two populations: the first comprises $80 \%$ of the system with $\langle[\mathrm{Fe} / \mathrm{H}]\rangle=-1.8$, the second, with the remaining $20 \%$, has $\langle[\mathrm{Fe} / \mathrm{H}]\rangle=-1.3$. From color-magnitude diagram studies, Lee et al. (1999) and Pancino et al. (2000) claim further, more metal-rich, minority populations.

\section{Timescale for cluster enrichment}

The relative abundances of the s-process elements offer the important opportunity of constraining the period over which cluster enrichment occurred. The work of Smith et al. $(1995,2000)$ is of critical importance. From a study of $[\mathrm{Rb} / \mathrm{Zr}]$ the latter authors show that $1.5-3 \mathrm{M}_{\odot}$ AGB stars have played a role in enriching the system. That is, cluster enrichment proceeded over a period of 2-3 Gyr. Claims of color-magnitude diagram morphology signatures consistent with an age spread of this order have also been reported (Hilker \& Richtler 1999; Hughes \& Wallerstein 2000).

${ }^{1}$ Superimposed on the primordial behavior of $\mathrm{Mg}$ one also sees the deep-mixing signature of $\mathrm{Mg}$ depletion. 
The abundance patterns discussed here, when coupled with kinematic information, place further constraints on the manner in which the cluster formed. This lies, however, somewhat outside the terms of reference of the present forum. The interested reader is referred to the work of Norris et al. (1997) where this matter is discussed.

\section{On-going main sequence investigation}

Spectroscopic work to date in $\omega$ Cen has centered on objects brighter than the horizontal branch. I conclude with a brief comment on a medium-resolution multi-fiber investigation of main sequence stars. Using the Two Degree Field facility on the Anglo-Australian Telescope, Cannon, Croke, Da Costa, Gibson, and the present author have obtained spectra of some 200 main sequence cluster members having signal-to-noise $\sim 30$ and resolving power 1600 . The (calcium) abundance spread described above is clearly seen in these data. Among the sample, one remarkable star stands out in which there is an enormous enhancement of the Sr II lines at 4077 and $4215 \AA$. Preliminary model atmospheric analysis yields $[\mathrm{Sr} / \mathrm{Fe}] \sim 2.0$ for this object, adding strong support to the earlier conclusions concerning cluster enrichment by AGB stars.

Acknowledgments. It is a pleasure to acknowledge collaborations over a long period with my colleagues at Mount Stromlo, from which I have benefited enormously - M. S. Bessell, G. S. Da Costa, and K. C. Freeman - and many enjoyable and challenging discussions with $\mathrm{A}$. W. Rodgers.

\section{References}

Cannon, R. D. \& Stobie, R. S. 1973, MNRAS, 162, 207

Hilker, M. \& Richtler, T. 1999, astro-ph/9910370

Hughes, J. \& Wallerstein, G. 2000, AJ, 119, 1225

Lee, Y.-W., Joo, J.-M., Sohn, Y.-J., Rey, S.-C., Lee, H.-C., \& Walker, A. R. 1999, Nature, 402,55

Norris, J. E. \& Da Costa, G. S. 1995a, ApJ, 441, L81

Norris, J. E. \& Da Costa, G. S. 1995b, ApJ, 447, 680

Norris, J. E., Freeman, K. C., \& Mighell, K. J. 1996, ApJ, 462, 241

Norris, J. E., Freeman, K. C., Mayor, M., \& Seitzer, P. 1997, ApJ, 487, L187

Pancino, E., Ferraro, F. R., Bellazzini, M., Piotto, G., \& Zoccali, M. 2000, ApJ, 534, L83

Persson, S. E., Frogel, J. A., Cohen, J. G., Aaronson, M., \& Matthews, K. 1980, ApJ, 235, 452

Smith, V. V., Cunha, K., \& Lambert, D. L. 1995, AJ, 110, 2827

Smith, V. V., Suntzeff, N. B., Cunha, K., Gallino, R., Busso, M., Lambert, D. L., \& Straniero, O. 2000, AJ, 119, 1239

Suntzeff, N. B. \& Kraft, R. P. 1996, AJ, 111, 1913

Woolley, R. v. d. R. et al. 1966, Roy. Obs. Ann., No. 2 